\title{
Acute Unilateral Pulmonary Edema in Non-Cardiac Settings
}

\author{
Jad Omran ${ }^{\mathrm{a}}$, Kanupriya Jain ${ }^{\mathrm{a}}$, Sudarshan Balla ${ }^{\mathrm{b}}$, Ramez Sunna ${ }^{\mathrm{c}, \mathrm{d}}$
}

\begin{abstract}
Unilateral pulmonary edema is an uncommon manifestation of pulmonary edema. We report a case of unilateral pulmonary edema in a 45-year-old woman with chronic kidney disease, hypertension and diabetes who presented after a motor vehicle collision and was down with the left lung in dependent position. She was found to have a left lung infiltrate on the chest computed tomography (CT) scan. Resolution of radiological findings after hemodialysis pointed toward pulmonary edema. The patient did not have any pre-existing parenchymal lung abnormality; therefore the cause of pulmonary edema was ascribed to dependent position.
\end{abstract}

Keywords: Pulmonary edema; Lung infiltrates; Dependent position

\section{Introduction}

Pulmonary edema caused by fluid overload is usually bilateral and symmetric [1]. Cardiopulmonary diseases may cause atypical findings such as unilateral or lobar edema [2]. Unilateral pulmonary edema is rare and may occur due to focal imbalance of Starling mechanism (between hydrostatic pressure and plasma oncotic pressure) caused by ab-

Manuscript accepted for publication May 19, 2014

${ }^{a}$ Department of Internal Medicine, University of Missouri-Columbia, One Hospital Drive, Columbia 65201, MO, USA

${ }^{\mathrm{b}}$ Department of Cardiovascular Medicine, University of Missouri-

Columbia, One Hospital Drive, Columbia 65201, MO, USA

'Department of Pulmonary and Critical Care Medicine, University of

Missouri-Columbia, One Hospital Drive, Columbia 65201, MO, USA

${ }^{\mathrm{d}}$ Corresponding Author: Ramez Sunna, Division of Pulmonary and

Critical Care Medicine, University of Missouri Health Care, One

Hospital Drive, Columbia 65201, MO, USA.

Email: sunnar@health.missouri.edu

doi: http://dx.doi.org/10.14740/jmc1808w normal pulmonary structure or other hemodynamic factors [3]. However, when unilateral pulmonary edema happens without any pre-disposing pulmonary abnormality, it is very uncommon [2].

\section{Case Report}

A 45-year-old African-American female with past medical history significant for end-stage renal disease, hypertension, type 2 diabetes, obstructive sleep apnea (on home oxygen therapy) and hyperlipidemia was admitted to emergency department after having motor vehicle accident. Patient ended up landing on her left side and stayed in that position for 30 min until she was recovered by emergency medical services. In the emergency department, the patient denied chest pain, shortness of breath or cough. She was afebrile and hemodynamically stable. Her oxygen saturation was $100 \%$ with 2 LPM via nasal cannula. Her physical exam was notable

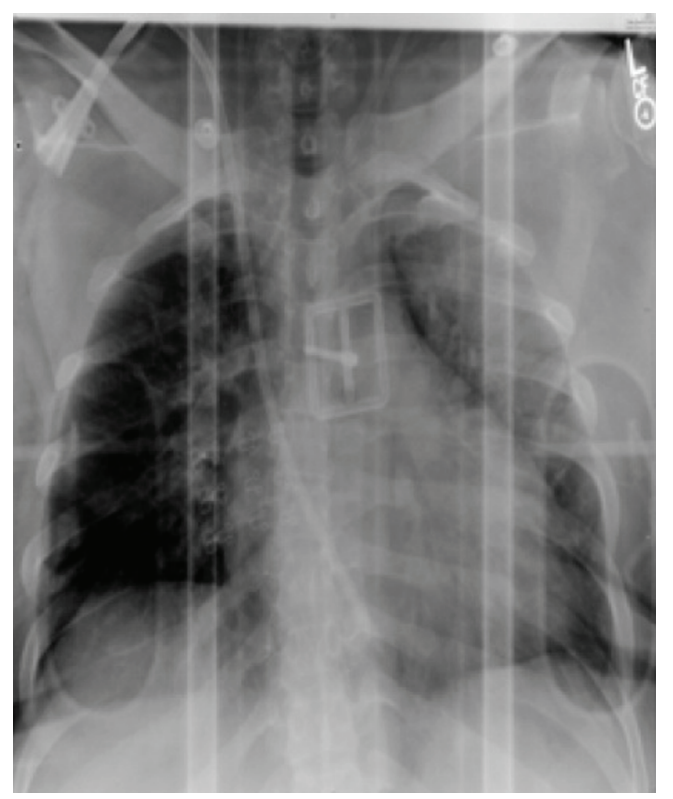

Figure 1. Chest X-ray on admission showing left perihilar opacities. 


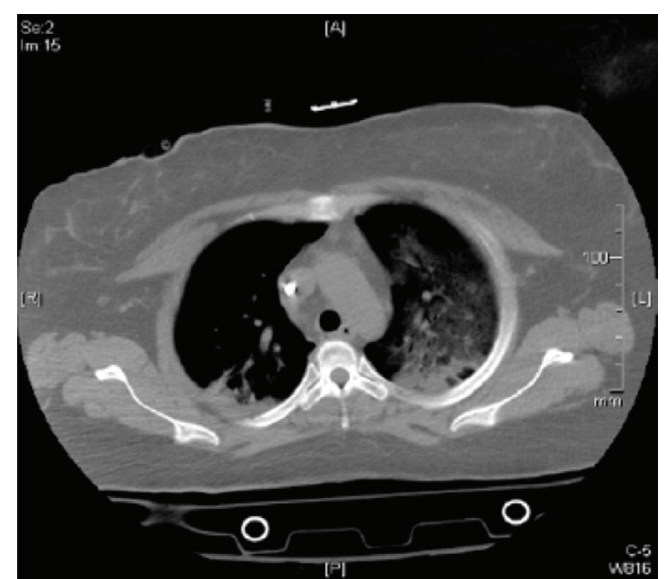

Figure 2. CT of chest on admission showing left-sided ground glass opacities.

for mildly decreased breath sounds on the left lung. There was no chest wall tenderness, appreciable murmur or peripheral edema. Labs showed a total leucocyte count 12,200/ $\mathrm{mm}^{3}$, hemoglobin $12 \mathrm{gm} \%$, platelets 276,000 , sodium 138 $\mathrm{mmol} / \mathrm{L}$, potassium $4.8 \mathrm{mmol} / \mathrm{L}$, glucose $121 \mathrm{mg} / \mathrm{dL}$, BUN $48 \mathrm{mg} / \mathrm{dL}$ and creatinine $10 \mathrm{mg} / \mathrm{dL}$. Chest radiograph (Fig. 1) showed stable mild cardiomegaly and left perihilar air space opacities with no pneumothorax or effusion. Computed tomography (CT) of chest (Fig. 2) showed diffuse ground glass opacities on the left side.

Pulmonary medicine was consulted, as there was concern of hypoxia and increased oxygen requirements. A diagnosis of unilateral pulmonary edema was made based on physical exam findings of unilateral crackles along with the radiographic findings. The patient mentioned that she had missed her dialysis session the previous day; therefore she

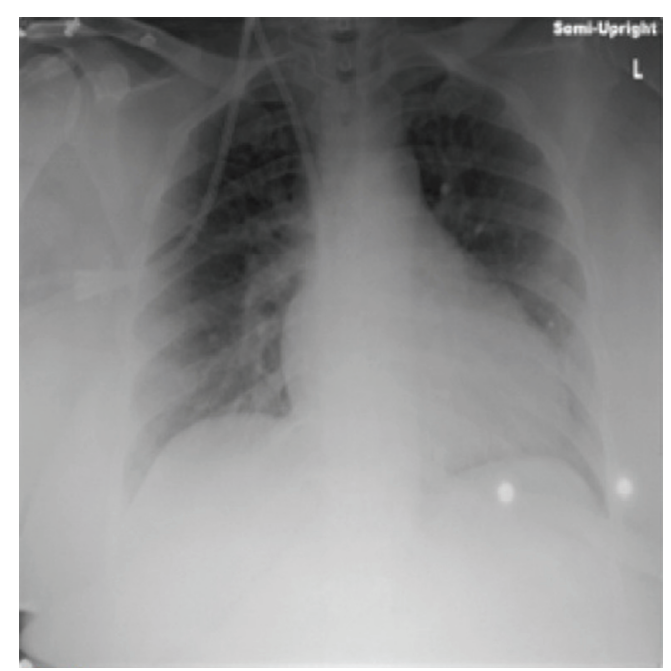

Figure 3. Chest $\mathrm{X}$-ray on the next day showing improvement in the left perihilar opacities.

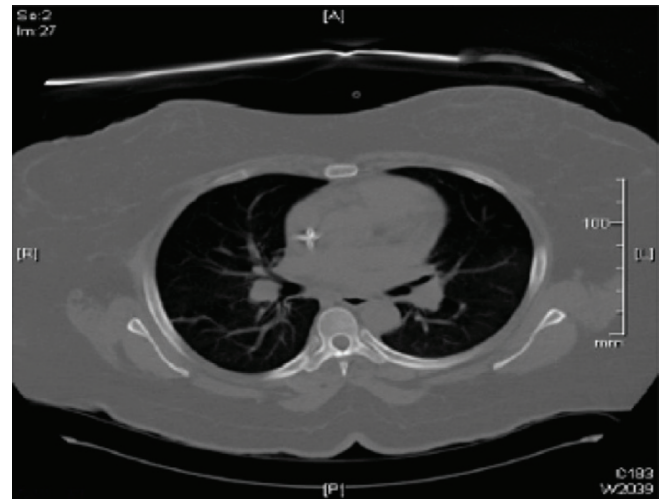

Figure 4. CT of chest on the next day showing resolution of the prior described left lung infiltrates.

underwent one hemodialysis session in our facility. On the next day, repeated chest X-ray (Fig. 3) and CT of chest (Fig. 4) showed resolution of the lung infiltrates and the patient was discharged home 1 day later.

\section{Discussion}

Acute unilateral pulmonary edema is an unusual clinical entity that often presents an interesting and confusing diagnostic challenge. Few case reports have described severe mitral regurgitation and eccentric mitral regurgitant jet as causes of unilateral pulmonary edema [4]. Other cases of unilateral pulmonary edema had different etiology including rapid evacuation of pleural fluid or air, causing re-expansion pulmonary edema [2]. Patients with chronic kidney disease and fluid overload can develop pulmonary edema which is usually bilateral and a result of extracellular fluid accumulation and reduction in plasma oncotic pressure often due to hypoalbuminaemia which can be seen in chronic kidney disease [5]. In our case, there was no evidence of mitral regurgitaion on exam. Prior echocardiograms did not reveal any evidence of valvular heart disease, and there was no structural abnormality of the pulmonary parenchyma. Based on the history, it was determined that the cause of pulmonary edema was dependent on the patient's decubitus position with her concomitant missing dialysis the day before. The increase in hydrostatic pressure in the dependent lung parenchyma is due to increased circulating intravascular volume and also to gravity. Dependent lung parenchyma has a relative hyperperfusion and hypoventilation, especially in our case context of volume overload may result in pulmonary edema [6]. The immediate resolution of pulmonary edema with dialysis further supports this pathogenesis. The differential diagnosis of pulmonary infiltrate is vast and unilateral pulmonary edema is one rare cause. Fluid overload related to ESRD and CHF should be considered in the differential diagnosis of unilateral pulmonary edema. Prompt recognition and therapy can 
avoid invasive procedures and inappropriate management.

\section{References}

1. Fleischner FG. The butterfly pattern of acute pulmonary edema. Am J Cardiol. 1967;20(1):39-46.

2. Calenoff L, Kruglik GD, Woodruff A. Unilateral pulmonary edema. Radiology. 1978;126(1):19-24.

3. Gluecker T, Capasso P, Schnyder P, Gudinchet F, Schaller MD, Revelly JP, Chiolero R, et al. Clinical and radiologic features of pulmonary edema. Radiographics. 1999;19(6):1507-1531; discussion 1532-1503.

4. Attias D, Mansencal N, Auvert B, Vieillard-Baron A, Delos A, Lacombe P, N'Guetta R, et al. Prevalence, characteristics, and outcomes of patients presenting with cardiogenic unilateral pulmonary edema. Circulation. 2010;122(11):1109-1115.

5. Pierson DJ. Respiratory considerations in the patient with renal failure. Respir Care. 2006;51(4):413-422.

6. Leeming BW. Gravitational edema of the lungs observed during assisted respiration. Chest. 1973;64(6):719-722. 